\title{
LA SUBORDINATION
}

La subordination peut être envisagée d'un point de vue purement syntaxique (subordination du ler degré (1), qui sera dite partielle/ du 2ème degré ou totale), ou sous l'angle morpho-syntaxique, le seul qui nous intéresse ici, dans le cadre de la subordination totale.

Sur le plan morpho-syntaxique, il y a lieu de distinguer deux sortes de subordination: la subordination translative, marquée par un mot translatif, et la subordination modale, marquée par le mode du verbe subordonné. Ces deux marques concurrentielles peuvent être associées corrélativement dans la subordination mixte ou ambivalente. Enfin, la subordination peut, théoriquement, n'être pas marquée du tout, comme c'est le cas de la subordination partielle dans Hôtel Dieu. Ces quatre cas de figures peuvent être rassemblés dans la matrice suivante:

\begin{tabular}{|l|c|c|c|c|}
\hline subordination & 1 & 2 & 3 & 4 \\
\hline translative & + & - & + & - \\
\hline modale & - & + & + & - \\
\hline
\end{tabular}

\section{LA SUBORDINATION TRANSLATIVE}

La subordination translative s'opère au moyen d'une conjonction de subordination ou d'un pronom/adjectif/adverbe relatif/interrogatif. En latin, lorsque, dans la subordonnée, le choix est possible entre indicatif et subjonctif, autrement dit lorsque l'opposition modale y est sémantiquement distinctive, le subjonctif ne saurait avoir de fonction subordonnante:

Roma sic appellata est quod a Romulo condita est.

"Romme fut ainsi appelée parce qu'elle fut fondée par Romulus" (cause objective) (2).

Romulus Remum fratrem interfecit quod per ludibrium novae urbis moenia transiluisset. 
"Romulus tua son frère parce que (selon lui) il avait sauté par dérision au-dessus des murailles de la nouvelle ville“ (2).

La subordination peut être annoncée/rappelée dans la principale/ dans la subordonnée par un corrélat général ou particulier ("antécédent" du pronom relatif). Situé dans la principale, le corrélat joue le rôle de translatif surordonant. Le dénivellement syntaxique est alors doublement marqué: vers le haut, dans la principale, et vers le bas, dans la subordonnée:

Ut sementem feceris, ita metes (Cicéron, De oratore).

"Tu récoltera comme tu auras semé" (3)

Ita est ici corrélat général anaphorique.

Id, quod constituerant facere conantur, ut exeant... (César, De bello Gallico, 1.5.1.)

“Ils entreprennent de faire ce qu'ils avaient décidé, à savoir de sortir...“ (4)

Id est ici corrélat-antécédent de quod et corrélat (cataphorique) de $u t$, comme dans:

Non is uir est, ut/qui... sentiat (Cicéron, Pro L. Valerio Flacco)

"Il n'est pas un homme à comprendre/tel qu'il comprenne" (4).

Silua, ubi/in qua sumus pulcherrima est.

"La forêt où nous sommes est très belle"

Silua est ici corrélat-antécédent particulier, ce qui ne l'empêche pas d'être surordonnant.

L'insertion de l'antécédent particulier dans la subordonnée substitue à sa fonction surordonnante une fonction subordonnante d'appoint, marquée par l'accord en cas avec le relatif:

Epistola, quam mihi misisti, mihi fuit gratissima

Quam mihi misisti epistolam, ea fuit mihi gratissima.

"La lettre que tu m'a écrite m'a fait grand plaisir" (5)

Ainsi doublement marqué dans la subordonnée, le dénivellement syntaxique se voit en outre renforcé par la présence, dans la principale, d'un corrélat surordonnant (ea). La subordination mixte (translative et modale: cf. infra) constitue un autre cas de dénivellement syntaxique doublement marqué dans la subordonnée. 


\section{SUBORDINATION MODALE}

En latin, la subordination peut n'être exprimée que par le mode du verbe de la subordonnée. Le latin possède trois modes subordonnants: un mode personnel, le subjonctif, et deux modes impersonnels, l'infinitif et le participe. Il y a donc lieu de distinguer la subordination subjonctive, qui est personnelle, et les subordinations infinitive et participiale, qui sont impersonnelles.

\subsection{La subordination modale personnelle: la subordination subjonctive}

La "grammaire essentielle du latin" (6) présente ainsi la subordination modale subjonctive: "Après un certain nombre de verbes et de locutions verbales, la proposition complétive au subjonctif peut n'être introduite par aucune conjonction de subordination; on dit qu'il y a simple parataxe (d'un mot grec signifiant juxtaposition); en d'autres termes, la complétive au subjonctif est une proposition au subjonctif de souhait ou d'ordre, simplement juxtaposée au verbe introducteur. " Or il y a contradiction entre les termes de "juxtaposition" ou "parataxe" qui impliquent deux propositions indépendantes, et de "complétive", nécessairement subordonnée à une principale. C'est donc faute d'avoir reconnu la valeur subordonnante du subjonctif que certains grammairiens parlent ici de parataxe. En fait, il y aurait lieu de distinguer entre juxtaposition, critère purement formel, et parataxe, critère fonctionnel, opposé à subordination ou hypotaxe. C'est ce que semble faire implicitement M. Lavency lorsqu'il écrit (7): “D'autres fois, il n'y a pas de mot subordonnant: la subordination peut alors être marquée à la fois par le cas du nom sujet (Acc. ou Abl. et non N.) et par le mode du verbe prédicat (infinitif ou participe, et non mode personnel); elle peut enfin, mais beaucoup plus rarement, être signalée par la seule juxtaposition et par le mode (subjonctif) du verbe.

Voici quelques exemples de subordination purement subjonctive, et donc purement modale: on notera qu'il s'agit toujours de subordonnées complétives (complément d'objet direct ou second actant, selon la terminologie de L. Tesnière):

Uolo tu ad me scribas. "Je veux que tu m'écrives.“ (8)

Perturbantur, copiasne ducere... an... an ... praestaret (César, De bello Gallico, 4.14.2).

"Dans le désarroi, ils se demandent s'il vaut mieux conduire les troupes... ou... ou...“ (9).

Et, avec un verbe principal impersonnel:

Haec faciat oportet. "A lui de faire cela" (10) 


\subsection{Subordination modale impersonnelle}

\subsubsection{Subordination infinitive}

Dans la proposition infinitive, c'es: la désinence d'infinitif qui fait fonction de subordonnant:

Sine puerum dormire. "Laisse l'enfant dormir"

Le sujet de la proposition infinitive est généralement à l'accusatif. Le sujet d'une proposition principale ou indépendante étant au nominatif, on a vu dans l'accusatif sujet de la proposition infinitive une marque de subordination (Lavency, cf supra). En réalité, si le sujet à l'accusatif est bien subordonné au verbe de la subordonnée, il est sous la dépendance du verbe principal, nécessairement transitif. La désinence d'accusatif apparaît, ainsi comme une résurgence de la proposition principale dans la subordonnée:
Sine -um puer- dorm-ire
(niveau de la principale)
(niveau de la subordonnée)

Vue sous cet angle, la désinence d'accusatif sert de corrélat surordonnant à celle d'infinitif, située dans la subordonnée.

Avec un verbe principal impersonnel, le sujet de la proposition infinitive peut être au datif:

Si nobis... perire hodie necesse est. (Tacite, Historiae, 1.29.4)

"S'il nous faut périr aujourd'hui" (11)

Sous la dépendance et dans le prolongement du verbe principal, ce datif, résurgence de la principale dans la subordonnée, est corrélatif de la désinence d'infinitif. A ce titre, et donc au même titre que l'accusatif sujet d'une proposition infinitive, il surordonne la principale à la proposition subordonnée par l'infinitif.

Quant à l'infinitif, forme semi-nominale du verbe, il est, de par la fonction nominale que lui confère cette forme, subordonné paradigmatique au verbe dont il est la projection. Cette subordination paradigmatique de l'infinitif au verbe dont il procède prête à la désinence d'infinitif une valeur subordonnante dans le syntagme. Par ailleurs, la fonction nominale de l'infinitif fait que cet infinitif ne peut guère être considéré comme surordonné à son sujet, auquel il est en quelque sorte apposé. La proposition infinitive apparaît ainsi comme une proposition aplatie, dans laquelle les rapports hiérarchiques entre verbes et actants sont plus ou moins affaiblis. De ce fait, le verbe de la proposition infinitive est quelque peu ambigu: n'étant pas tout à fait un verbe - puisque non assujetti à la catégorie de personne -, il n'est pas vraiment surordonné à son sujet, n'étant pas tout à fait un nom - puisque affranchi des 
catégories de cas, de genre et de nombre - il ne lui est pas vraiment apposé non plus.

La proposition infinitive n'étant ni verbale ni nominale ne constitue pas plus une translation du 2ème degré qu'une translation du ler degré. Elle est donc neutre sous le rapport de l'opposition syntaxique /partiel/:/total/, c'est-à-dire qu'elle n'est ni un simple syntagme à l'intérieur d'une proposition, ni une proposition complète à l'intérieur d'une phrase. Par son sujet à l'accusatif, rattaché directement au verbe de la principale, la proposition infinitive est bien moins autonome que la proposition conjonctive ou même relative. Situé à l'intersection de la principale et de la subordonnée, ce sujet à l'accusatif ne peut être comparé qu'au "relatif à antécédent incorporé", également intersectionnel, et de plus symétrique, par sa fonction, de l'accusatif sujet de la proposition infinitive. En effet, celui-ci est objet partiel comme le pronom relatif à antécédent incorporé est sujet partiel du verbe de la principale:

Credo Caesarem caecum fuisse

Quign'avance pas recule

On ne peut pas plus écrire: ${ }^{*}$ Credo Caesarem (objet partiel) que *Qui recule (sujet partiel).

A la différence du latin, le russe, le slovène et la français n'admettent pas d'autres propositions infinitives que celles dont l'accusatif sujet pourrait à lui seul servir de complément d'objet intégral du verbe de la principale:

russe: ostav' ego spat'

slovène: pusti ga spati

français: "laisse-le dormir"

parce que ostav' ego, pusti ga, "laisse-le" sont corrects.

\subsubsection{La subordination participiale}

Le sujet de la proposition participiale est au cas exigé par sa fonction adverbale/adnominale dans la phrase. Les propositions participiales adverbales sont actancielles (nominales)/circonstancielles (adverbiales). On n'envisagera ici que les propositions participiales actancielles.

Le sujet d'une proposition participiale elle-même sujet du verbe principal est naturellement au nominatif. Le sujet d'une proposition participiale complétive directe/indirecte est à l'accusatif/au datif: 
Senatus querentes eos non sustinuit. (Tite-Live, 31. 13.14)

"Le Sénat ne résista pas à leurs plaintes" (12).

Reuertentibus inde eis Tiburtes portas clausere. (Tite-Live, 7.9.1)

"A leur retour, Tibur leur ferma ses portes" (13)

Le paradigme pronominal A. eos/D. eis est dépendant de la valence des verbes sustinuit, clausere. Sujets des propositions participiales querentes eos, reuertentibus inde eis, les pronoms eos/eis n'en font pas moins respectivement partie des principales (eos) non sustinuit, (eis) Tiburtes portas clausere: ils sont donc à l'intersection des propositions principales et subordonnées. Mais le participe évacue la catégorie spécifiquement verbale de personne. Par ailleurs, l'accord avec le sujet se fait en genre, nombre et cas, catégories nominales. Or, seule celle de nombre est commune au verbe et au participe. C'est donc autour du nombre que le syntagme participial oscille entre la perspective verbale d'un syntagme total (par translation du 2ème degré) et la perspective nominale d'un syntagme partiel (par translation du ler degré):

syntagme verbal avec surordination verbale:

syntagme nominal avec subordination adjectivale:<smiles>CC(C)C(C)(C)C(C)(C)C</smiles><smiles>ClC(Cl)(Cl)C=CC1CCCCC1</smiles>

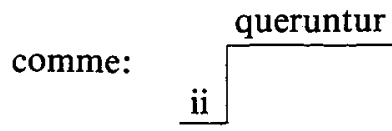

comme: $\stackrel{\text { ii }}{\text { infelices }}$

En fin de compte, la subordination relève non seulement de l'opposition bipolaire /total/:/partiel/, mais aussi de son déploiement en statuts neutre et ambivalent, selon la matrice suivante:

\begin{tabular}{|l|c|c|c|c|}
\hline subordination & 1 & 2 & 3 & 4 \\
\hline partielle & + & - & + & - \\
\hline totale & - & + & + & - \\
\hline & $\begin{array}{l}\text { subordination } \\
\text { partielle: à } \\
\text { l'intérieur } \\
\text { de la propo- } \\
\text { sition ou de } \\
\text { la phrase } \\
\text { simple }\end{array}$ & $\begin{array}{l}\text { subordination } \\
\text { totale: à } \\
\text { l'intérieur } \\
\text { de la phrase } \\
\text { complexe: } \\
\text { subordination } \\
\text { translative } \\
\text { (conjonctive, } \\
\text { relative, in- } \\
\text { terrogative) } \\
\text { et/ou modale } \\
\text { personnelle }\end{array}$ & $\begin{array}{l}\text { subordination } \\
\text { ambivalente: } \\
\text { à la fois } \\
\text { partielle et } \\
\text { totale: } \\
\text { subordination } \\
\text { participiale }\end{array}$ & $\begin{array}{l}\text { subordination } \\
\text { neutre (ni } \\
\text { partielle ni } \\
\text { totale): } \\
\text { subordination } \\
\text { infinitive }\end{array}$ \\
\cline { 3 - 5 } & & $\begin{array}{l}\text { subordination modale imperson- } \\
\text { nelle }\end{array}$ & \\
\hline
\end{tabular}




\section{REFERENCES BIBLIOGRAPHIQUES}

1. Tesnière L., Éléments de syntaxe structurale, Paris 1959, Klincksieck.

2. Gaillard J., Cousteix J., Grammaire essentielle du latin, Paris 1976, Scodel, p. 206.

3. Lavency M., Usus, description du latin classique en vue de la lecture des auteurs, Paris-Gembloux 1985, Duculot. Par. 395, p. 252.

4. Gaffiot F., Dictionnaire illustré latin-français, Paris 1934.

5. Lavency M., op. cit., par. 366, p. 233.

6. Gaillard J., Cousteix J., op. cit. p. 199.

7. Lavency M., op. cit., par. 328, p. 209. (Non souligné dans le texte.)

8. Gaillard J., Cousteix J., op. cit. p. 199.

9. Gaffiot F., op. cit.: praesto.

10. Lavency M., op. cit., par. 341, p. 219.

11. Oudot J., Syntaxe latine, Strasbourg 1964, Publications de la Faculté des Lettres de l'Université, p. 270.

12. Ibid., p. 292.

13. Ibid., p. 293.

Povzetek

PODREDNOST

Glede na skladnjo je podrednost lahko parcialna (prvostopenjska), totalna, ambivalentna (deležniška podrednost) ali nevtralna (nedoločniška podrednost). Glede na morfo-sintaktično ravnino je totalna podrednost lahko translacijska, naklonsko osebna (subjunktivna /lat.: konjunktivna podrednost), naklonsko neosebna (nedoločniška, deležniška podrednost). Skladenjski razmik je lahko dvojno označen: splošni in posebni korelati ("antecedenti") veznikov in relativnih zaimkov so iz tega ustvarili korelativno nadredno vrednost podredne funkcije vezniških besed, katere se na njih povratno navezujejo ali pa jih napovedujejo. V nedoločniškem stavku imajo obrazila akuzativnega in dativnega subjekta (ponoven pojav glavnega stavka $v$ odvisnem) ravno tako nadredno vrednost. Torej obstaja korelacija med sklonskim obrazilom subjekta, ki ne more biti v nominativu, in obrazilom nedoločnika, kar navsezadnje pomeni dvojno označenost: nadredno in podredno. 